\title{
Family History and Functional Outcome in Korean Stroke Patients: A Preliminary Study
}

\author{
Hee Jung Park, MD', Tae Uk Kim, MD, PhD ${ }^{1}$, Jung Keun Hyun, MD, PhD ${ }^{1,2,3}$, Jung Yoon Kim, MD, PhD ${ }^{1,4}$
}

\author{
${ }^{1}$ Department of Rehabilitation Medicine, Dankook University College of Medicine, Cheonan; \\ ${ }^{2}$ Department of Nanobiomedical Science \& WCU Research Center, Dankook University, Cheonan; \\ ${ }^{3}$ Institute of Tissue Regeneration Engineering (ITREN), Dankook University, Cheonan; \\ ${ }^{4}$ Ewha Brain Institute, Ewha Womans University, Seoul, Korea
}

Objective To investigate the association of family history of stroke with functional outcomes in stroke patients in Korea.

Methods A case-control study was conducted. A total of 170 patients who were admitted to a rehabilitation unit were included. Risk factors for stroke such as age, sex, diabetes mellitus, hypertension, atrial fibrillation, smoking, high blood cholesterol and homocysteine level, obesity, and family history of stroke were taken into account. Stroke subtypes were the following: large vessel infarct, small vessel infarct, embolic infarct, subarachnoid hemorrhage, and intracranial hemorrhage. Stroke severity as assessed with the National Institutes of Health Stroke Scale (NIHSS), functional outcomes using the Korean version of the Modified Barthel index (K-MBI), Functional Independence Measurement (FIM), and cognitive function using the Korean version of Mini-Mental State Examination (K-MMSE) were assessed at admission and discharge.

Results Subjects with a family history of stroke were more likely to have an ischemic stroke (90.7\%) than were those without a family history (70.9\%). The K-MBI, FIM, NIHSS, and K-MMSE scores did not show significant differences between patients with or without family history.

Conclusion Family history of stroke was significantly associated with ischemic stroke, but not with functional outcomes. Other prognostic factors of stroke were not distributed differently between patients included in this study with or without a family history of stroke.

Keywords Stroke, Family history, Functional outcome, Prognosis, Ischemic stroke

Received April 24, 2015; Accepted July 18, 2015

Corresponding author: Jung Yoon Kim

Ewha Brain Institute, Ewha Womans University, 52, Ewhayeodae-gil, Seodaemun-gu, Seoul 03760, Korea

Tel: +82-2-3277-6559, Fax: +82-2-3277-6562, E-mail: schola21@gmail. com

(c) This is an open-access article distributed under the terms of the Creative Commons Attribution Non-Commercial License (http://creativecommons. org/licenses/by-nc/4.0) which permits unrestricted noncommercial use, distribution, and reproduction in any medium, provided the original work is properly cited.

Copyright $\odot 2015$ by Korean Academy of Rehabilitation Medicine

\section{INTRODUCTION}

Stroke is one of the major causes of impairment and mortality in developed countries. Rates of stroke survival with disability are variable worldwide, but several studies have reported predictors of post-stroke functioning to be patient age, stroke subtype, severity, location and size of stroke, and family history of stroke. Family history is a vital piece of medical information because family members 
partially share genes, environment, and lifestyles, which play significant roles in the development of stroke $[1,2]$. Also, knowledge of family history is an excellent starting point in investigating genetic factors in stroke, their effects on stroke risk, and prognoses.

Previous studies on family history usually focused on its role as a risk factor rather than as a prognostic factor [2-6]. There has been no consensus as to whether family history is a prognostic factor [2-6]. This is partially because other risk factors for stroke, such as diabetes mellitus, hypertension, and hyperhomocysteinemia, also have familial connections, so the effects of each are difficult to tease apart.

As family history is closely related to genetics, individuals of different ethnicities may have different risk factors and prognoses. In fact, slight discrepancies have been previously identified between races [4,6-9].

One study reported that a family history of stroke was related to ischemic stroke subtype and to poor functional status, as assessed using the modified Rankin Scale, at discharge [7], while no significant relationship to outcome was reported in another study [9].

To our knowledge, studies on the association of family history of stroke and functional outcomes in Asian populations are scarce. One article from China reports only an indirect association between family history and metabolic syndrome, and discusses the effects of metabolic syndrome on stroke [10].

The aim of this study was to obtain preliminary data on the relation of family history of stroke with functional outcomes and stroke severity in stroke patients in Korea.

\section{MATERIALS AND METHODS}

\section{Study subjects}

A retrospective case-control study was performed. Electronic medical records of stroke patients who were admitted to the rehabilitation unit of Dankook Universitiy Hospital from May 2008 to June 2013 were screened. Microsoft SQL Server 2014 Express (Microsoft, Redmond, WA, USA) was used to extract data from the hospital's electronic medical record database.

A total of 184 patients were diagnosed with stroke during the period in question, but 14 were excluded because rehabilitative treatments were discontinued due to other medical conditions. Thus, data from 170 patients was an- alyzed. Participants were divided into two groups: those with a family history of stroke, and those with no family history of stroke-FHx(+) vs. $\mathrm{FHx}(-)$, respectively.

\section{Baseline characteristics}

Demographic characteristics such as age and gender were recorded. Patients or their family members were asked whether any first-degree relative had experienced a stroke. A first-degree relative is a family member who shares a significant amount of genetic material with a particular individual. First-degree relatives include parents, offspring, and siblings [11].

Risk factors for stroke including hypertension, diabetes mellitus, atrial fibrillation, and hypercholesterolemia were also recorded at the time of admission. Hypertension was identified based on a previous diagnosis or current treatment with antihypertensive drugs. Atrial fibrillation was evaluated with electrocardiography upon admission. Hypercholesterolemia was diagnosed when serum cholesterol levels exceeded $250 \mathrm{mg} / \mathrm{dL}$, and hyperhomocysteinemia when serum levels were greater than $15.39 \mu \mathrm{mol} / \mathrm{L}$. A poorly controlled glucose level was defined as serum hemoglobin Alc ( $\mathrm{HbAlc})>6.5 \%$. Body mass index was calculated using measurements of body weight and height taken at the time of admission. A patient was marked positive for smoking if he/she had been smoking for more than 5 years. The total length of stay in rehabilitation (in days) was recorded as a measure of treatment intensity. Stroke types were classified into 5 categories using the Harvard Cooperative Stroke Registry: large vessel infarct, small vessel infarct, embolic infarct, subarachnoid hemorrhage (SAH), and intracranial hemorrhage (ICH) [12].

\section{Outcome measures}

Stroke severity was measured using the National Institutes of Health Stroke Scale (NIHSS) score at admission and discharge [13].

Functional outcomes were assessed at admission and discharge using the Korean version of the Modified Barthel Index (K-MBI) and the Functional Independence Measure (FIM) [14,15]. Cognitive functions were evaluated using the Korean version of Mini-Mental State Examination (K-MMSE) at admission and discharge [16]. 
Table 1. Baseline characteristics of study subjects

\begin{tabular}{|lccc}
\hline & \multicolumn{2}{c}{ Family history } & \multirow{2}{*}{ p-value } \\
\cline { 2 - 3 } & $\mathbf{( + )}$ & $(-)$ & 0.844 \\
\hline Age (yr) & $64.1 \pm 11.7$ & $62.6 \pm 13.4$ & 0.425 \\
Gender (male:female) & $23: 20$ & $59: 68$ & 0.359 \\
\hline Hypertension & $32 / 43(74.4)$ & $85 / 127(67.0)$ & 0.055 \\
\hline Diabetes mellitus & $19 / 43(44.2)$ & $36 / 127(28.3)$ & 0.494 \\
\hline Atrial fibrillation & $1 / 43(2.3)$ & $6 / 127(4.7)$ & 0.036 \\
\hline Hypercholesterolemia & $6 / 42(14.3)$ & $6 / 127(4.7)$ & 0.077 \\
\hline Smoking & $9 / 43(20.9)$ & $45 / 127(35.4)$ & 0.299 \\
\hline Hyperhomocysteinemia & $9 / 37(24.3)$ & $13 / 80(16.3)$ & 0.111 \\
\hline HbAlc $(>6.5 \%)$ & $18 / 39(46.2)$ & $28 / 89(31.5)$ & 0.362 \\
\hline Body mass index $\left(>25 \mathrm{~kg} / \mathrm{m}^{2}\right)$ & $15 / 43(34.9)$ & $35 / 127(27.6)$ & 0.101 \\
\hline Length of stay (day) & $45.7 \pm 24.9$ & $41.1 \pm 27.7$ & \\
\hline Vas & & & \\
\hline
\end{tabular}

Values are presented as mean \pm standard deviation or number (\%).

Table 2. Distribution of stroke subtype according to family history

\begin{tabular}{lcc}
\hline \multirow{2}{*}{ Stroke subtype } & \multicolumn{2}{c}{ Family history } \\
\cline { 2 - 3 } & $(+)$ & $(-)$ \\
\hline Ischemic stroke & & \\
Small vessel infarct & $20 / 43(46.5)$ & $41 / 127(32.3)$ \\
Large vessel infarct & $6 / 43(14.0)$ & $30 / 127(23.6)$ \\
Embolic infarct & $13 / 43(30.2)$ & $19 / 127(15.0)$ \\
Hemorrhagic stroke & & \\
\hline Intracranial hemorrhage & $4 / 43(9.3)$ & $27 / 127(21.3)$ \\
\hline Subarachnoid hemorrhage & $0 / 43(0)$ & $10 / 127(7.9)$ \\
\hline
\end{tabular}

Values are presented as number (\%).

\section{Statistical analysis}

All statistical analyses were performed using STATA/ MP 13.0 for Windows (StataCorp LP, College Station, TX, USA). Baseline characteristics and outcome measures between the two groups (with or without family history of stroke), were compared by a chi-square test using categorical variables, and by the Mann-Whitney U test using continuous variables. Changes between status at admission and discharge were analyzed using the Wilcoxon signed-rank test and Mann-Whitney U test. Normality was measured with the Shapiro-Wilk W test. Statistical significance was set at $p$-value $<0.05$. Data are given as mean \pm standard deviation.
Table 3. Distribution of stroke subtype (ischemic vs. hemorrhagic) according to family history

\begin{tabular}{llll}
\hline & \multicolumn{2}{c}{ Family history } & p-value \\
\cline { 2 - 3 } & $(+)$ & $(-)$ & \\
\hline Ischemic stroke & $39 / 43(90.7)$ & $90 / 127(70.9)$ & 0.009 \\
Hemorrhagic stroke & $4 / 43(9.3)$ & $37 / 127(29.1)$ & \\
\hline Values are presented as number (\%).
\end{tabular}

\section{RESULTS}

\section{Baseline characteristics}

A total of 170 subjects ( 82 men and 88 women, aged $63.0 \pm 13.0$ years) were included in this study. Forty-three patients $(25.3 \%)$ reported that at least one first-degree relative had experienced a stroke (FHx(+) group). The $\mathrm{FHx}(+)$ and $\mathrm{FHx}(-)$ groups did not show significant differences in age, gender, known risk factors for stroke, total length of stay in rehabilitation; there was a significant difference in hypercholesterolemia (Table 1) such that there was a significantly higher incidence of hypercholesterolemia in the $\mathrm{FHx}(+)$ group (Table 1 ).

The distributions of stroke subtypes were significantly different between the groups ( $\mathrm{p}=0.011$ by chi-square test). Stroke subtypes in the $\mathrm{FHx}(+)$ group were $46.5 \%$ small vessel infarct, $30.2 \%$ embolic infarct, $14.0 \%$ large vessel infarct, and 9.3\% intracranial hemorrhage. No individual in the $\mathrm{FHx}(+)$ group had a subarachnoid hemorrhage (Table 2). Stroke subtypes in the FHx(-) group were $32.3 \%$ 
Table 4. Outcome measures in patients with or without a family history of stroke

\begin{tabular}{|c|c|c|c|}
\hline & \multicolumn{2}{|c|}{ Family history } & \multirow{2}{*}{ p-value ${ }^{a}$} \\
\hline & $(+)$ & $(-)$ & \\
\hline \multicolumn{4}{|l|}{ NIHSS } \\
\hline Admission & $5.7 \pm 4.4$ & $5.6 \pm 4.4$ & 0.881 \\
\hline Discharge & $3.6 \pm 3.5$ & $3.5 \pm 2.6$ & 0.872 \\
\hline p-value ${ }^{\text {b) }}$ & $<0.001$ & $<0.001$ & \\
\hline Gain & $2.1 \pm 2.6$ & $2.1 \pm 2.6$ & 0.857 \\
\hline \multicolumn{4}{|l|}{ K-MBI } \\
\hline Admission & $50.1 \pm 26.1$ & $48.0 \pm 25.7$ & 0.628 \\
\hline Discharge & $69.6 \pm 26.8$ & $70.0 \pm 25.2$ & 0.922 \\
\hline p-value ${ }^{b)}$ & 0.001 & 0.001 & \\
\hline Gain & $19.5 \pm 12.7$ & $22.1 \pm 17.5$ & 0.675 \\
\hline \multicolumn{4}{|l|}{ FIM } \\
\hline Admission & $73.0 \pm 25.0$ & $69.9 \pm 25.1$ & 0.489 \\
\hline Discharge & $89.9 \pm 28.2$ & $89.0 \pm 25.2$ & 0.847 \\
\hline p-value ${ }^{b)}$ & $<0.001$ & $<0.001$ & \\
\hline Gain & $16.9 \pm 11.1$ & $19.1 \pm 16.0$ & 0.658 \\
\hline \multicolumn{4}{|l|}{ K-MMSE } \\
\hline Admission & $21.9 \pm 8.1$ & $20.1 \pm 7.8$ & 0.210 \\
\hline Discharge & $23.8 \pm 8.1$ & $23.1 \pm 6.3$ & 0.602 \\
\hline p-value ${ }^{\text {b) }}$ & $<0.001$ & $<0.001$ & \\
\hline Gain & $1.9 \pm 2.1$ & $3.0 \pm 4.2$ & 0.144 \\
\hline
\end{tabular}

Values are presented as mean \pm standard deviation. NIHSS, National Institutes of Health Stroke Scale; KMBI, Korean version of Modified Barthel Index; FIM, Functional Independence Measure; K-MMSE, Korean version of Mini-Mental State Examination.

${ }^{\text {a) }} \mathrm{p}$-value, between groups.

${ }^{b)} \mathrm{p}$-value, between time.

small vessel infarct, $23.6 \%$ large vessel infarct, $21.3 \%$ intracranial hemorrhage, $15.0 \%$ multiple embolic infarct, and $7.9 \%$ subarachnoid hemorrhage (Table 2). When classified into ischemic vs. hemorrhagic stroke, ischemic stroke was more common in the $\mathrm{FHx}(+)$ group than in the $\mathrm{FHx}(-)$ group ( $90.7 \%$ vs. $70.9 \%$, respectively; $\mathrm{p}=0.009$ by chi-square test) (Table 3 ).

\section{Outcome measures}

The K-MBI, FIM, K-MMSE, and NIHSS scores were not significantly different between the two groups at admission or discharge. There were significant changes in all outcome variables between admission and discharge, representing functional improvements and neurologic recovery (Table 4). Family history of stroke, $\mathrm{FHx}(+)$ or $\mathrm{FHx}(-)$, did not have a significant effect on the gains in the NIHSS, K-MBI, FIM, and K-MMSE scores (Table 4).

Even when stroke outcome was divided into favorable (K-MBI $\geq 90$ ) or unfavorable (K-MBI $<90$ ), a family history of stroke had no significant effect on outcomes (favorable vs. poor outcomes, 31:12 in the $\mathrm{FHx}(+)$ group and 87:40 in the $\mathrm{FHx}(-)$ group; $\mathrm{p}=0.659$ by a chi-square test).

Because no subject in the $\mathrm{FHx}(+)$ had a $\mathrm{SAH}$, it was impossible to evaluate the relationship between family history and functional outcome in SAH. Considering all other stroke subtypes, recovery, as assessed by the NIHSS, K-MBI, FIM, and K-MMSE, was not significantly different between patients with and without a family history of stroke (data not presented).

\section{DISCUSSION}

Our results are consistent with previous reports that showed no significant association of family history with stroke outcome [9]. This study contributes to the ongoing controversy concerning whether or not a family history of stroke can be considered a prognostic factor [7,9]; a family history of stroke has already been well established as a risk factor of stroke occurrence [2-6]. We found that stroke subtypes differed depending on family history, as ischemic strokes were more common in individuals in the $\mathrm{FHx}(+)$ group. This result is also consistent with previous findings $[2,7]$. A previous study showed that a family history of stroke was associated with small vessel infarcts or large vessel infarcts. However, a family history of stroke was more closely associated with small vessel infarcts and embolic infarcts in our study. Although hypertension is attributable to environmental factors such as smoking, diet and exercise, etc., genetics also strongly influence the prevalence of hypertension [17]. This may explain why small vessel infarcts were more common in patients with a family history of stroke. A family history of atrial fibrillation has been shown to increase the risk of atrial fibrillation [18]. Genome-wide association studies have found 27 genetic variants that are associated with an increased risk of myocardial infarction [19]. Increased risks of atrial fibrillation and myocardial infarction together may affect the incidence of embolic infarcts.

In a previous study, a history of stroke in a first-degree relative was associated with poor functional outcomes 
(modified Rankin Scale score $\geq 2$ at discharge) [7]. In contrast, functional outcomes, as evaluated using primarily the K-MBI and the FIM in our study, did not differ in patients with and without a family history. To eliminate measurement effects of outcome variables (e.g., dichotomous vs. continuous variables), the K-MBI scores were divided into favorable or poor functional outcomes. Still, no differences were found between the $\mathrm{FHx}(+)$ and $\mathrm{FHx}(-)$ groups.

The above inconsistencies could be caused by differences in demographics or baseline characteristics. Genetic, national, social, and environmental factors could all exert effects on functional outcomes in stroke patients. Ethnic and genetic traits specific to Koreans could contribute to the discrepancy between the previous results [7] and those presented here. There have been reports of ethnicity affecting stroke risk and type $[8,20]$, so it is reasonable to think that the usefulness of family history as a prognostic factor may differ between the Korean population and other ethnic populations.

Some limitations of this study need to be discussed. The sample size was small, and was limited to one university hospital. However, distributions of gender, stroke subtypes, and ratio of positive family history of stroke were quite similar to those used in previous studies $[2,21,22]$. This suggests that the characteristics of our sample did not deviate greatly from those of the population of stroke patients as a whole. Another limitation was the absence of long-term follow-ups. Nevertheless, the length of stay of $42.3 \pm 27.0$ days does cover the critical period for stroke rehabilitation [23].

The sample size that would have been able to detect significant differences between favorable outcomes (favorable outcome $27.9 \%$ in $\mathrm{FHx}(+)$ group and $31.5 \%$ in $\mathrm{FHx}(-)$ group), with a test power of $80 \%$, significance level of 0.05 , and effect size of 0.5 , was calculated to be 5,056 subjects. It would be impossible to obtain a sample size of this magnitude in one hospital or by a few individual researchers. Initiating a multi-center trial or enlarging the stroke rehabilitation registry would be necessary to conduct research with sufficient statistical power.

Evaluating prognostic factors very soon after admission to the rehabilitation unit could be vital in the effort to predict outcomes, manage rehabilitation programs precisely, and inform patients and family members. A largecohort study using the data from a few stroke registries, such as the Brain Rehabilitation Registry or the Korea Stroke Registry, would be needed to fully elucidate the relationship between family history of stroke and functional outcome of stroke in Korea. Data from our study can be used to suggest the necessary sample size, and to determine feasible outcome measures.

In conclusion, a family history of stroke was significantly associated with the incidence of ischemic stroke, but not with functional outcomes in stroke patients. Other risk factors and prognostic factors were not different between patients with or without a family history of stroke.

\section{CONFLICT OF INTEREST}

No potential conflict of interest relevant to this article was reported.

\section{REFERENCES}

1. Graffagnino C, Gasecki AP, Doig GS, Hachinski VC. The importance of family history in cerebrovascular disease. Stroke 1994;25:1599-604.

2. Jerrard-Dunne P, Cloud G, Hassan A, Markus HS. Evaluating the genetic component of ischemic stroke subtypes: a family history study. Stroke 2003;34:1364-9.

3. Jood K, Ladenvall C, Rosengren A, Blomstrand C, Jern C. Family history in ischemic stroke before 70 years of age: the Sahlgrenska Academy Study on Ischemic Stroke. Stroke 2005;36:1383-7.

4. Aycock DM, Kirkendoll KD, Coleman KC, Clark PC, Albright KC, Alexandrov AW. Family history of stroke among African Americans and its association with risk factors, knowledge, perceptions, and exercise. J Cardiovasc Nurs 2015;30:E1-6.

5. Debette S, Goeggel Simonetti B, Schilling S, Martin JJ, Kloss M, Sarikaya H, et al. Familial occurrence and heritable connective tissue disorders in cervical artery dissection. Neurology 2014;83:2023-31.

6. O'Neill SM, Rubinstein WS, Wang C, Yoon PW, Acheson LS, Rothrock N, et al. Familial risk for common diseases in primary care: the Family Healthware Impact Trial. Am J Prev Med 2009;36:506-14.

7. Lisabeth LD, Smith MA, Brown DL, Uchino K, Morgenstern LB. Family history and stroke outcome in a bi-ethnic, population-based stroke surveillance study. BMC Neurol 2005;5:20. 
8. Morgenstern LB, Smith MA, Lisabeth LD, Risser JM, Uchino K, Garcia N, et al. Excess stroke in Mexican Americans compared with non-Hispanic Whites: the Brain Attack Surveillance in Corpus Christi Project. Am J Epidemiol 2004;160:376-83.

9. Harmsen P, Lappas G, Rosengren A, Wilhelmsen L. Long-term risk factors for stroke: twenty-eight years of follow-up of 7457 middle-aged men in Goteborg, Sweden. Stroke 2006;37:1663-7.

10. Mi D, Zhang L, Wang C, Liu L, Pu Y, Zhao X, et al. Impact of metabolic syndrome on the prognosis of ischemic stroke secondary to symptomatic intracranial atherosclerosis in Chinese patients. PLoS One 2012; 7:e51421.

11. Genetics Home Reference. First-degree relative [Internet]. Bethesda: Genomics Home Reference, National Library of Medicine; 2015 [cited 2015 Nov 10]. Available from: http://ghr.nlm.nih.gov/glossary=firstdegreerelative.

12. Mohr JP, Caplan LR, Melski JW, Goldstein RJ, Duncan GW, Kistler JP, et al. The Harvard Cooperative Stroke Registry: a prospective registry. Neurology 1978;28: 754-62.

13. Goldstein LB, Samsa GP. Reliability of the National Institutes of Health Stroke Scale: extension to nonneurologists in the context of a clinical trial. Stroke 1997;28:307-10.

14. Jung HY, Park BK, Shin HS, Kang YK, Pyun SB, Paik NJ, et al. Development of the Korean version of Modified Barthel Index (K-MBI): multi-center study for subjects with stroke. J Korean Acad Rehabil Med 2007;31:283-
97.

15. Hamilton BB, Laughlin JA, Fiedler RC, Granger CV. Interrater reliability of the 7-level functional independence measure (FIM). Scand J Rehabil Med 1994;26:115-9.

16. Park JH. Modification of the mini-mental state examination for use in the elderly in a non-western society. Part 1: Development of Korean version of mini-mental state examination. Int J Geriatr Psychiatry 1990;5:381-7.

17. Lifton RP, Gharavi AG, Geller DS. Molecular mechanisms of human hypertension. Cell 2001;104:545-56.

18. Palatini P. Parental atrial fibrillation as a risk factor for atrial fibrillation in offspring. JAMA 2004;292:1174-5.

19. O'Donnell CJ, Nabel EG. Genomics of cardiovascular disease. N Engl J Med 2011;365:2098-109.

20. Markus HS, Khan U, Birns J, Evans A, Kalra L, Rudd AG, et al. Differences in stroke subtypes between black and white patients with stroke: the South London Ethnicity and Stroke Study. Circulation 2007;116:2157-64.

21. Adams HP Jr, Bendixen BH, Kappelle LJ, Biller J, Love BB, Gordon DL, et al. Classification of subtype of acute ischemic stroke: definitions for use in a multicenter clinical trial (Trial of Org 10172 in Acute Stroke Treatment). Stroke 1993;24:35-41.

22. Polychronopoulos P, Gioldasis G, Ellul J, Metallinos IC, Lekka NP, Paschalis C, et al. Family history of stroke in stroke types and subtypes. J Neurol Sci 2002; 195:117-22.

23. Murphy TH, Corbett D. Plasticity during stroke recovery: from synapse to behaviour. Nat Rev Neurosci 2009; 10:861-72. 